\title{
Generalized Local Binary Patterns for Texture Classification
}

\author{
$\mathrm{Li} \mathrm{Liu}^{1}$ \\ dreamliu2010@gmail.com \\ Paul Fieguth ${ }^{2}$ \\ pfieguth@uwaterloo.ca \\ Gangyao Kuang ${ }^{1}$ \\ kuangyeats@vip.sina.com
}

\author{
${ }^{1}$ Electronic Science and Engineering \\ National University of Defense \\ Technology \\ Changsha, Hunan, China 410073 \\ ${ }^{2}$ System Design Engineering Dept. \\ University of Waterloo \\ Waterloo, Ontario, Canada N2L 3G1
}

\begin{abstract}
This paper presents a novel approach for texture classification, generalizing the wellknown local binary patterns (LBP). In the proposed approach, two different and complementary types of features are extracted from local patches, based on pixel intensities and differences. Inspired by the LBP approach, two intensity-based and two difference-based descriptors are developed. All four descriptors have the same form as the conventional LBP codes, thus they can be readily combined to form joint histograms to represent textured images. The proposed approach is computationally simple and is training-free: there is no need to learn a texton dictionary and no tuning of parameters. Extensive experimental results on two challenging texture databases (Outex and KTHTIPS2b) show that the proposed approach significantly outperforms the classical LBP approach and other state-of-the-art methods with a nearest neighbor classifier.
\end{abstract}

\section{Introduction}

Texture classification is a fundamental issue in computer vision and image processing, playing a significant role in a wide range of applications that includes medical image analysis, remote sensing, object recognition, document analysis, environment modeling, content-based image retrieval and many more [1].

Recently, the orderless Bag-of-Words (BoW) approach has proven extremely popular and successful in texture classification tasks. Robust and discriminative local texture descriptors and global statistical histogram characterization have supplied complementary components toward the BoW feature extraction of texture images. It is generally agreed that the local descriptors play a much important role, therefore have received considerable attention during the last decade and numerous local descriptors have been proposed [2, 3, 5, 6, 7, 8, 9].

Due to its impressive computational efficiency and good texture discriminative property, the dense LBP descriptor [5] has gained considerable attention since its publication [12], and has already been used in many other applications $[13,14,15] .{ }^{1}$ Despite the great success of

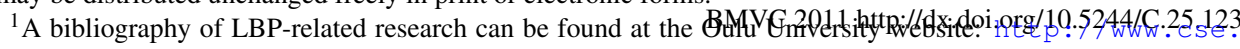




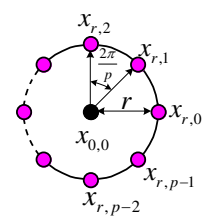

Figure 1: A central pixel $x_{0,0}$ and its $p$ circularly and evenly spaced neighbors $\left\{x_{r, i}\right\}_{i=0}^{p-1}$ on radius $r$.

LBP in computer vision and pattern recognition, the conventional LBP operator comes with the following disadvantages and limitations: long histograms sensitive to image rotation, the small spatial area of support, a loss of local textural information, and noise sensitivity.

To be sure, researchers have made efforts to address LBP limitations and have proposed LBP variants. In terms of locality, [16] proposes to extract global features from the Gabor filter responses as a complementary descriptor. To recover from the loss of information, local image contrast has been introduced by Ojala et al. [5] as a complementary measure, and better performance has been reported therein. Moreover, Guo et al. [17] include the magnitudes of local differences and claim better performance.

With regards to noise robustness, Ojala et al. [5] introduce the concept of uniform and rotation invariant patterns. Ahonen et al. introduce soft histograms [22]. Tan and Triggs [23] propose local ternary patterns. In addition, very recently, Heikkilä et al. [18] exploit circular symmetric LBP (CS-LBP) for local interest region description, and Chen et al. present a WLD descriptor by including orientation information as a robust descriptor [19].

The LBP approach is based on the assumption that the local differences of the central pixel and its neighbors are independent of the central pixel itself. In practice this independence is not warranted, and the value of the central pixel may also be significant, leading to the questions central to this paper: Is explicitly modeling the joint distribution of the central pixel and its neighbors an advantage or disadvantage, and how to effectively include the missing between-scale information so that better texture classification can be achieved?

Motivated by [3] and [5], in this paper we propose a simple, yet very powerful and novel texture descriptor to generalize the conventional LBP approach. In the proposed approach, the LBP coding strategy is applied to two different but complementary types of features: pixel intensities and intensity differences. The common LBP coding strategy allows the descriptors to be fused, and significantly improved classification results are achieved.

The paper is organized as follows. Section 2 starts with a review of the classical LBP approach and then details the derivation of the proposed descriptors and classification scheme. In Section 3, we verify the proposed approach with extensive experiments on popular texture datasets and comparisons with various state-of-the-art texture classification techniques. Section 4 provides concluding remarks and possible extensions.

\section{Proposed Descriptors}

\subsection{A Brief Review of LBP}

The LBP method was first proposed by Ojala et al. [5] to encode the pixel-wise information in textured images. Images are probed locally by sampling greyscale values at a central point $x_{0,0}$ and $p$ points $x_{r, 0}, \ldots, x_{r, p-1}$ spaced equidistantly around a circle of radius $r$ centered at 
$x_{0,0}$, as shown in Fig. 1. Formally,

$$
L B P_{p, r}=\sum_{n=0}^{p-1} s\left(x_{r, n}-x_{0,0}\right) 2^{n}, \quad s(x)=\left\{\begin{array}{l}
1, x \geq 0 \\
0, x<0
\end{array}\right.
$$

The gray values of neighbors which do not lie precisely in a pixel location may be estimated by interpolation.

Given an $N \times M$ image $\mathbf{I}$, let $L B P_{p, r}(i, j)$ be the identified LBP pattern of each pixel $(i, j)$, then the whole texture image is represented by a histogram vector $\underline{\boldsymbol{h}}$ of length $K$ :

$$
\underline{\boldsymbol{h}}(k)=\sum_{i=1}^{N} \sum_{j=1}^{M} \delta\left(L B P_{p, r}(i, j)-k\right)
$$

where $0 \leq k \leq K-1$, and $K=2^{p}$ is the number of all the LBP codes. Feature $h$ has attractive properties: gray-scale invariance, low complexity, few parameters, and satisfactory discriminating power.

However, the basic LBP operator produces rather long histograms ( $2^{p}$ distinct values), and it becomes an intractable problem to estimate $\underline{\boldsymbol{h}}$ due to the overwhelming dimensionality of $\underline{\boldsymbol{h}}$ with large $p$. Moreover, it is easy to realize that due to the way LBP numbers are created, they are very sensitive to noise. One improvement suggested by Ojala et al. [5] is to consider only the so-called "uniform" pattern $L B P_{p, r}^{r i u 2}$, merging nonuniform patterns directly into one pattern:

$$
L B P_{p, r}^{r i w 2}= \begin{cases}\sum_{n=0}^{p-1} s\left(x_{r, n}-x_{0,0}\right), & \text { if } U\left(L B P_{p, r}\right) \leq 2 \\ p+1, & \text { otherwise }\end{cases}
$$

where

$$
U\left(L B P_{p, r}\right)=\sum_{n=0}^{p-1}\left|s\left(x_{r, n}-x_{0,0}\right)-s\left(x_{r, \bmod (n+1, p)}-x_{0,0}\right)\right|
$$

The superscript riu2 denotes the rotation invariant "uniform" patterns that have $U$ values at most 2. Therefore, mapping from $L B P_{p, r}$ to $L B P_{p, r}^{r i u 2}$ results in only $p+2$ distinct groups of patterns, leading to a much shorter histogram representation.

\subsection{Intensity-based Descriptors}

Inspired by Markov Random Field (MRF) models, we propose to use only local neighborhood distributions, similar to ideas of Varma and Zisserman [3]. In MRF modeling, the probability of a central pixel $\mathbf{I}\left(x_{c}\right)$ depends only on its neighborhood $\mathscr{N}\left(x_{c}\right)$. In this paper we explicitly model the joint distribution of a central pixel and its neighbors, in order to test the significance of the conditional probability distribution for classification.

Inspired by the coding strategy of LBP, we define the following NI-LBPdescriptor (see also Fig. 2):

$$
N I-L B P_{p, r}=\sum_{n=0}^{p-1} s\left(x_{r, n}-\mu\right) 2^{n}, \quad \text { where } \quad \mu=\frac{1}{p} \sum_{n=0}^{p-1} x_{r, n}
$$

Similar to $L B P_{p, r}^{r i u 2}$, the rotation invariant version of $N I-L B P$, denoted by $N I-L B P_{p, r}^{r i u}$, can also be defined to achieve rotation invariant classification. 


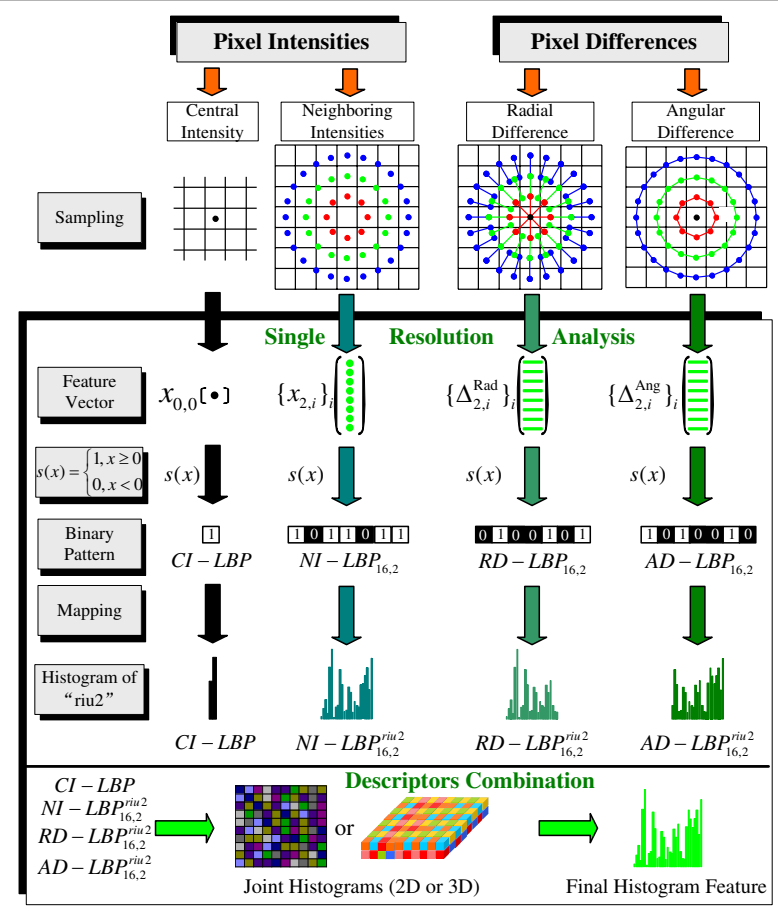

Figure 2: Overview of the proposed approach.

Next, the local contrast measure proposed by Ojala et al. [5] is defined as

$$
V A R_{p, r}=\frac{1}{p} \sum_{n=0}^{p-1}\left(x_{r, n}-\mu\right)^{2}, \quad \text { where } \quad \mu=\frac{1}{p} \sum_{n=0}^{p-1} x_{r, n}
$$

$N I-L B P_{p, r}$ and $V A R_{p, r}$ capture similar types of texture information, with a few differences. Clearly, $N I-L B P_{p, r}$ is independent of gray scale, whereas $V A R_{p, r}$ is not. $V A R_{p, r}$ is not affected by rotation, but $N I-L B P_{p, r}$ is, by default. Finally, whereas $N I-L B P_{p, r}$ is discrete, $V A R_{p, r}$ produces continuous values which need to be quantized, which requiring training to determine a threshold value, and there exist issues in setting the number of bins.

To maintain consistency with the standard binary coding strategy, the central pixels intensity is discretized as $C I-L B P=s\left(x_{0,0}-\mu_{I}\right)$, relative to $\mu_{I}$, the mean of image $\mathbf{I}$.

\subsection{Difference-based Descriptors}

We propose two different descriptors, Radial Difference Local Binary Pattern and Angular Difference Local Binary Pattern (denoted as RD-LBP and AD-LBP respectively and as illustrated in Fig. 2):

$$
R D-L B P_{p, r, \delta}=\sum_{n=0}^{p-1} s\left(\Delta_{\delta, n}^{\mathrm{Rad}}\right) 2^{n}, \quad A D-L B P_{p, r, \delta}=\sum_{n=0}^{p-1} s\left(\Delta_{\delta, n}^{\mathrm{Ang}}\right) 2^{n}
$$

where $\delta$ is an integer, and $\Delta_{\delta, n}^{\mathrm{Rad}}=x_{r, n}-x_{r-\delta, n}$ is the radial difference computed with given radial displacement $\delta$, and $\Delta_{\delta, n}^{\mathrm{Ang}}=x_{r, n}-x_{r, \bmod (n+\delta, p)}$ is the angular difference computed with 


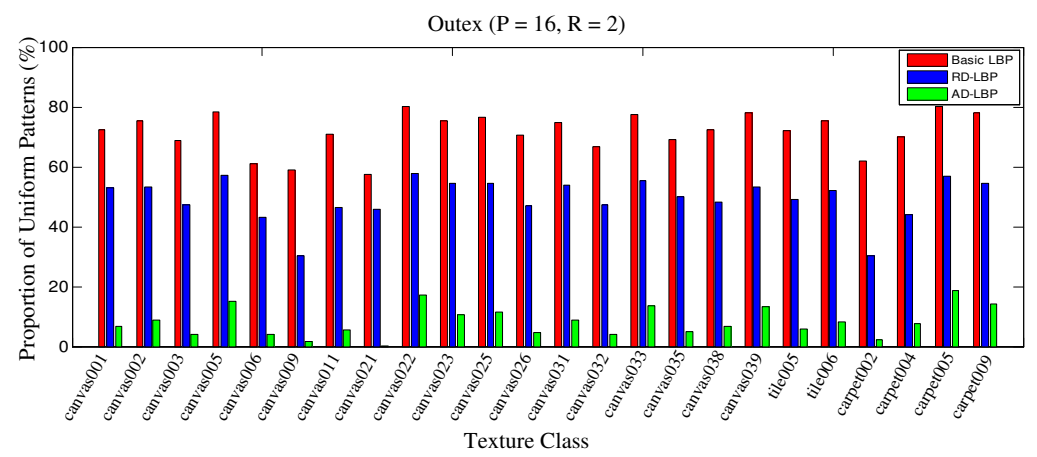

Figure 3: Comparing the proportions (in \%) of "Uniform" patterns for each texture in Outex for three methods: LBP, RD-LBP and AD-LBP, with $P=16, R=2$.

given angular displacement $\delta(2 \pi / p)$, and where $x_{r, n}$ and $x_{r, \bmod (n+\delta, p)}$ correspond to the gray values of pairs of pixels of $\delta$ equally spaced pixels on a circular radius $r$. We can see that when $\delta=p / 2$, our descriptor $A D-L B P_{p, r, p / 2}$ is equivalent to the the CS-LBP descriptor proposed by Heikkilä [18] for local interesting region description.

The uniform patterns represent meaningful and fundamental characteristics of the local texture structures; examining the proportions of the uniform patterns for LBP, RD-LBP and AD-LBP clearly demonstrated that the proportions of the uniform patterns of AD-LBP were too small and inadequate to provide a reliable and meaningful description of texture images, as illustrated in Fig. 3. Consequently we focus on the RD-LBP descriptor.

Next, there are two ways to combine the NI-LBP and RD-LBP codes: the concatenation of individual histograms, or jointly calculating a two dimensional histogram, represented as NI-LBP / RD-LBP. In this paper, we prefer the latter approach, which has also been used by Varma and Zisserman [3] and Guo et al. [17], and has been shown to produce better results. Following [5], we use only joint distributions of operators that have the same $(p, r)$ values, although nothing would prevent us from using joint distributions of operators computed at different neighborhoods.

\subsection{MultiResolution Analysis and Classification}

The proposed descriptors described above are extracted from single resolution with a circularly symmetric neighbor set of $p$ pixels placed on a circle of radius $r$ (see Fig. 2). It is generally agreed that multiresolution analysis is beneficial for texture classification. Obviously, by altering $(p, r)$, we can realize operators for any quantization of the angular space and for any spatial resolution. Motivated by the idea of [5], we conduct the multiresolution analysis by combining the information provided by multiple descriptors of varying $(p, r)$. The histogram feature vector of multiresolusion analysis is obtained by concatenating the histograms from multiple resolution analysis realized with different $(p, r)$. To perform the texture classification, nearest neighbor classifier (NNC) is used in this paper. The samples are then classified according to their normalized histogram feature vectors $\underline{\boldsymbol{h}}_{i}$ and $\underline{\boldsymbol{h}}_{j}$, using $\chi^{2}$ distance metric $\chi^{2}\left(\underline{\boldsymbol{h}}_{i}, \underline{\boldsymbol{h}}_{j}\right)=\frac{1}{2} \sum_{k} \frac{\left[\underline{\boldsymbol{h}}_{i}(k)-\underline{\boldsymbol{h}}_{j}(k)\right]^{2}}{\underline{\boldsymbol{h}}_{i}(k)+\underline{\boldsymbol{h}}_{j}(k)}$ (the same distance metric used in $\left.[2,3,21,24]\right)$. 
Table 1: Summary of texture datasets used in our experiments. Experiment \# 1

\begin{tabular}{|c|c|c|c|c|c|c|c|c|}
\hline $\begin{array}{l}\text { Texture } \\
\text { Dataset }\end{array}$ & $\begin{array}{l}\text { Texture } \\
\text { Classes }\end{array}$ & $\begin{array}{l}\text { Samples } \\
\text { per class }\end{array}$ & $\begin{array}{l}\text { Sample } \\
\text { Size }\end{array}$ & $\begin{array}{l}\text { Test } \\
\text { Suite }\end{array}$ & \begin{tabular}{|c|} 
Training \\
or Testing
\end{tabular} & $\begin{array}{l}\text { Number } \\
\text { of Angles }\end{array}$ & \begin{tabular}{c|} 
Illuminant \\
Used
\end{tabular} & $\begin{array}{l}\text { Samples } \\
\text { in Total }\end{array}$ \\
\hline \multirow[b]{2}{*}{ Brodatz } & \multirow[b]{2}{*}{16} & \multirow[b]{2}{*}{8} & \multirow[b]{2}{*}{$180 \times 180$} & \multirow{2}{*}{$\begin{array}{l}\text { Contrib_TC_00001 } \\
\text { (Problem 000-009) }\end{array}$} & Training & 1 & "inca" & $16(16 \times 1)$ \\
\hline & & & & & Testing & 9 & "inca" & $1008(16 \times 7 \times 9)$ \\
\hline \multirow{6}{*}{ Outex } & \multirow{6}{*}{24} & \multirow{6}{*}{20} & \multirow{6}{*}{$128 \times 128$} & \multirow{2}{*}{ Outex_TC_00010 } & Training & 1 & "inca" & $480(24 \times 20)$ \\
\hline & & & & & Testing & 8 & "inca" & $3840(24 \times 20 \times 8)$ \\
\hline & & & & \multirow{2}{*}{$\begin{array}{c}\text { Outex_TC_00012 } \\
\text { (Problem 000) }\end{array}$} & Training & 1 & "inca" & $480(24 \times 20)$ \\
\hline & & & & & Testing & 9 & "tl84" & $4320(24 \times 20 \times 9)$ \\
\hline & & & & \multirow{2}{*}{$\begin{array}{c}\text { Outex_TC_00012 } \\
\text { (Problem 001) }\end{array}$} & Training & 1 & "inca" & $480(24 \times 20)$ \\
\hline & & & & & Testing & 9 & "horizon" & $4320(24 \times 20 \times 9)$ \\
\hline & & & & \multicolumn{5}{|c|}{ Experiment \# 2} \\
\hline $\begin{array}{l}\text { Texture } \\
\text { Dataset }\end{array}$ & $\begin{array}{c}\text { Pose } \\
\text { Variation }\end{array}$ & $\begin{array}{l}\text { In-Plane } \\
\text { Rotation } \\
\end{array}$ & \begin{tabular}{|c|} 
Controlled \\
Illumination \\
\end{tabular} & $\begin{array}{c}\text { Scale } \\
\text { Variation }\end{array}$ & $\begin{array}{l}\text { Texture } \\
\text { Classes }\end{array}$ & $\begin{array}{c}\text { Sample } \\
\text { Size }\end{array}$ & $\begin{array}{l}\text { Samples } \\
\text { per class }\end{array}$ & $\begin{array}{l}\text { Samples } \\
\text { in Total }\end{array}$ \\
\hline KTH-TIPS $2 b$ & $\sqrt{ }$ & & $\sqrt{ }$ & $\sqrt{ }$ & 11 & $200 \times 200$ & 432 & $4752(432 \times 11)$ \\
\hline
\end{tabular}

Table 2: Abbreviations for the notations of methods.

\begin{tabular}{|c|c|c|c|}
\hline Name of Methods & Abbreviation & Name of Methods & Abbreviation \\
\hline $\begin{array}{l}L B P_{p, r}^{r i u 2} \\
L B P_{p, r}^{r i u 2} / V A R_{p, r}\end{array}$ & $\begin{array}{l}L B P \\
L B P / V A R\end{array}$ & $V A R_{p, r}$ & VAR \\
\hline $\begin{array}{l}N I-L B P_{p, r}^{r i u 2} \\
R D-L B P_{p, r}^{\text {riu } 2} / C I-L B P \\
N I-L B P_{p, r}^{\text {riu2 }} / R D-L B P_{p, r}^{r i u 2}\end{array}$ & $\begin{array}{l}N I \\
R D / C I \\
N I / R D\end{array}$ & $\begin{array}{l}R D-L B P_{p, r}^{r i u 2} \\
N I-L B P_{p, r}^{r i u 2} / C I-L B P \\
N I-L B P_{p, r}^{\text {riu2 }} / R D-L B P_{p, r}^{r i u 2} / C I\end{array}$ & $\begin{array}{l}R D \\
N I / C I \\
N I / R D / C I\end{array}$ \\
\hline
\end{tabular}

\section{Experimental Evaluation}

In this section, we demonstrate the performance of the proposed method with comprehensive experiments on five texture datasets, summarized in Table 1, which are derived from three popular publicly available texture databases: Brodatz [25], Outex [5], and KTHTIPS2b [21, 24].

Experimental \#1, presented in Section 3.2, aims at investigating the proposed approach for gray scale and rotation invariant texture classification, comparing our proposed descriptors with the classical LBP and VAR descriptors proposed in the original work by Ojala $e t$ al. [5] and other LBP based approaches [16, 17, 26]. Best scores achieved by our method are then compared with those reported by other state-of-the-art methods.

Experiment \#2, presented in Section 3.3, examines the classification performance of the proposed approach for a more realistic and challenging texture classification task: material categorization where each material consists of instances imaged from multiple different physical samples under different viewpoint, illumination and imaging distance, using the material database KTHTIPS2b [21, 24].

\subsection{Methods In Comparison}

To make the comparisons as meaningful as possible, we keep our experimental settings as similar as possible to [5]. The abbreviations for the proposed descriptors are summarized in Table 2. In all our experiments, each texture sample is normalized to be zero mean and unit standard deviation.

We compare with joint $L B P_{p, r}^{r i u 2} / V A R_{p, r}, L B P_{p, r}^{r i u 2}$ and $V A R_{p, r}$. We follow the experimental setup in [5] for these three descriptors. DLBP and NGF follow from [16]. CLBP [17] is training free, representing a local texture patch by its center pixel and the signs and magnitudes of the differences between center and neighborhood. VZ-MR8 [2, 3] and VZ-Joint [3] both require a time consuming universal texton dictionary learning stage. 
Table 3: Classification accuracies (\%) on Contrib_TC_00001, where training is done at just one rotation angle and the average accuracy over 10 angles. The results for $L B P, V A R$, and $L B P / V A R$ are quoted directly from the original paper by Ojala et al. [5].

\begin{tabular}{|c|c|c|c|c|c|c|c|c|c|c|c|c|c|}
\hline \multirow{2}{*}{ Method } & \multirow{2}{*}{$(\mathrm{p}, \mathrm{r})$} & \multirow[b]{2}{*}{ Bins } & \multicolumn{10}{|c|}{ Rotation Angle for Train } & \multirow[b]{2}{*}{ Average } \\
\hline & & & $0^{\circ}$ & $20^{\circ}$ & $30^{\circ}$ & $45^{\circ}$ & $60^{\circ}$ & $70^{\circ}$ & $90^{\circ}$ & $120^{\circ}$ & $135^{\circ}$ & $150^{\circ}$ & \\
\hline$\overline{L B P}$ & $(16$ & 18 & $\overline{96.2}$ & 99.0 & 98.6 & 98.9 & 98.5 & 99.1 & 97.6 & 98.6 & 98.7 & 97.5 & 98.3 \\
\hline$V A R$ & $(16,2)$ & 128 & 89.9 & 84.5 & 986.2 & 90.5 & 87.3 & 85.6 & 91.0 & 89.8 & 90.8 & 88.5 & 88.4 \\
\hline$L B P / V A R[5]$ & $1)+(16,2)+(24,3)$ & 864 & 100 & 99.7 & 99.5 & 99.8 & 99.6 & 99.7 & 99.8 & 99.6 & 99.8 & 99.9 & 99.7 \\
\hline \multirow{3}{*}{$N I$} & $\overline{(8,1)}$ & $\overline{10}$ & $\overline{65.4}$ & $\overline{85.5}$ & 81.3 & $\overline{76.6}$ & $\overline{\overline{77.0}}$ & $\overline{78.4}$ & $\overline{68.8}$ & $\overline{81.4}$ & $\overline{75.8}$ & $\overline{76.5}$ & $\overline{76.7}$ \\
\hline & $(16,2)$ & 18 & 87.6 & 95.2 & 92.3 & 93.6 & 89.4 & 96.0 & 88.9 & 91.3 & 93.4 & 90.1 & 91.8 \\
\hline & $(24,3)$ & 26 & 96.2 & 93.4 & 97.6 & 96.6 & 98.3 & 96.7 & 97.1 & 96.7 & 92.6 & 98.2 & 96.4 \\
\hline \multirow{3}{*}{$R D$} & $(8,1)$ & 10 & 68.8 & 86.4 & 84.4 & 76.0 & 84.9 & 84.4 & 70.2 & 84.1 & 76.1 & 84.7 & 80.0 \\
\hline & $(16,2)$ & 18 & 89.2 & 92.9 & 96.7 & 97.8 & 96.1 & 92.6 & 88.4 & 94.7 & 96.7 & 97.3 & 94.3 \\
\hline & $(24,3)$ & 26 & 87.6 & 90.6 & 98.2 & 90.8 & 96.5 & 93.8 & 89.5 & 98.6 & 89.5 & 94.2 & 92.9 \\
\hline \multirow{3}{*}{$R D / C I$} & $(8,1)$ & 20 & 87.1 & 94.7 & 94.3 & 88.6 & 95.9 & 95.1 & 85.8 & 94.8 & 90.3 & 95.0 & 92.2 \\
\hline & (1 & 36 & 92.7 & 94.6 & 96.8 & 97.3 & 98.4 & 95.6 & 91.8 & 99.4 & 96.7 & 98.6 & 96.2 \\
\hline & $(24,3)$ & 52 & 96.9 & 95.8 & 95.6 & 92.8 & 96.5 & 94.3 & 96.9 & 99.1 & 95.3 & 95.9 & 95.9 \\
\hline \multirow{3}{*}{$N I / C I$} & $(8,1)$ & 20 & 74.8 & 90.4 & 86.4 & 80.3 & 82.5 & 85.2 & 74.4 & 86.2 & 80.6 & 82.2 & 82.3 \\
\hline & $(16,2)$ & 36 & 95.6 & 99.2 & 98.8 & 98.0 & 98.2 & 99.4 & 93.8 & 98.3 & 96.9 & 97.4 & 97.6 \\
\hline & $(24,3)$ & 52 & 99.1 & 98.7 & 99.4 & 99.4 & 100 & 100 & 99.7 & 97.5 & 97.3 & 99.1 & 99.1 \\
\hline \multirow{3}{*}{$N I / R D$} & $(8,1)$ & 100 & 70.2 & 88.9 & 87.0 & 80.0 & 85.2 & 85.5 & 71.9 & 87.1 & 81.6 & 84.9 & 82.2 \\
\hline & $(16,2)$ & 324 & 100 & 100 & 100 & 100 & 100 & 100 & 100 & 100 & 100 & 100 & 100 \\
\hline & $(24,3)$ & 676 & $\overline{98.2}$ & 100 & 100 & 100 & 100 & $\overline{100}$ & 99.6 & $\overline{99.9}$ & $\overline{99.9}$ & $\overline{100}$ & $\overline{99.8}$ \\
\hline \multirow{3}{*}{$N I / R D / C I$} & $(8,1)$ & 200 & 78.1 & 94.5 & 92.2 & 91.1 & 93.0 & 92.0 & 76.2 & 92.4 & 91.8 & 92.6 & 89.4 \\
\hline & & 648 & 100 & 100 & 100 & 100 & 100 & 100 & 100 & 100 & 100 & 100 & 100 \\
\hline & $(24,3)$ & 1352 & $\overline{98.8}$ & 100 & 100 & 100 & $\overline{100}$ & $\overline{100}$ & 99.8 & 100 & $\overline{99.8}$ & $\overline{100}$ & $\overline{99.8}$ \\
\hline
\end{tabular}

Table 4: Classification accuracies $(\%)$ for all the three Outex test suites, where training was done at 0 angle and testing used 9 angles. The mean accuracy is the average over the three test suites. The results for $L B P, V A R$, and $L B P / V A R$ are quoted directly from the original paper by Ojala et al. [5].

\begin{tabular}{|c|c|c|c|c|c|c|c|c|c|c|c|c|}
\hline \multirow{2}{*}{ Test Suite } & \multicolumn{6}{|c|}{ Outex_TC_00012 } & \multirow{2}{*}{\multicolumn{3}{|c|}{$\frac{\text { Outex_TC_00010 }}{\text { "Inca" }}$}} & \multirow{2}{*}{\multicolumn{3}{|c|}{ Mean Accuracy }} \\
\hline & & $184^{\prime}$, & & & horizon & & & & & & & \\
\hline$(\mathrm{p}, \mathrm{r})$ & $(8,1)$ & $(16,2)$ & $(24,3)$ & $(8,1)$ & $(16,2)$ & $\overline{(24,3)}$ & $(8,1)$ & $(16,2)$ & $(24,3)$ & $(8,1)$ & $(16,2)$ & $(24,3)$ \\
\hline$\overline{L B P}[5]$ & 67.5 & 81.2 & 84.0 & 62.7 & 74.1 & 80.5 & 85.1 & 88.5 & 94.6 & 71.8 & 81.3 & 86.4 \\
\hline$V A R$ [5] & 64.3 & 67.1 & 62.6 & 64.7 & 72.5 & 68.9 & 91.2 & 90.7 & 86.2 & 73.4 & 76.8 & 72.6 \\
\hline$L B P / V A R$ [5] & 78.8 & 86.1 & 86.6 & 76.7 & 84.8 & 87.2 & 95.4 & 97.2 & 97.8 & 83.6 & 89.4 & 90.5 \\
\hline$\overline{N I}$ & 59.1 & 71.9 & 76.3 & 56.2 & 65.5 & 72.2 & 76.4 & 87.0 & 88.7 & 63.9 & 74.8 & 79.1 \\
\hline$R D$ & 67.0 & 77.4 & 76.8 & 63.1 & 72.3 & 72.1 & 81.0 & 86.6 & 89.7 & 70.4 & 78.8 & 79.5 \\
\hline $\mathrm{NI} / \mathrm{CI}$ & 76.5 & 88.6 & 88.9 & 77.4 & 89.4 & 84.6 & 89.9 & 96.4 & 95.7 & 81.3 & 91.5 & 89.7 \\
\hline$R D / C I$ & 87.9 & 91.9 & 86.1 & 88.3 & 91.5 & 82.3 & 95.2 & 95.9 & 93.7 & 90.7 & 93.1 & 87.4 \\
\hline$N I / R D$ & 79.0 & 96.2 & 95.2 & 80.8 & 95.2 & 92.2 & 88.9 & 98.7 & 98.8 & 82.9 & 96.7 & 95.4 \\
\hline$N I / R D / C I$ & 90.9 & 98.0 & 97.3 & 92.7 & 98.0 & 96.2 & 96.5 & 99.3 & 99.2 & 93.4 & 98.4 & 97.6 \\
\hline
\end{tabular}

\subsection{Experiment \#1}

Image Data and Experimental Setup. Test suite Contrib_TC_00001 was designated for rotation invariant texture classification, consists of 16 texture classes from the Brodatz database [25]. The experimental setup for this test suite is kept as same as [5].

Test suite Outex_TC_00010 contains 24 Outex texture classes with each class having 20 samples. The training and testing scheme for this test suite is the same as that for Contrib_TC_00001 but with nine different rotation angles.

Finally test suite Outex_TC_00012 was created by Ojalaet al. [5] for rotation and illumination invariant texture classification. Due to variations in illuminants, some texture samples have a large tactile dimension, which induce significant local gray-scale distortions, therefore Outex_TC_00012 is more challenging than Outex_TC_00010.

Experimental Results. Table 3 presents the results for our proposed descriptors on Contrib_TC_00001, comparing with the state-of-the-art methods from [5]. It is evident that the performance of the proposed $N I-L B P / R D-L B P$ and $N I-L B P / R D-L B P / C I-L B P$ descriptors are superior to that of $L B P / V A R$. Note that here we only consider single resolution for our descriptors; it may be argued that this test suite is too easy for texture classification, nevertheless our $100 \%$ classification scores demonstrate a very strong performance.

Next, Table 4 presents results for our proposed descriptors and those proposed in [5] on test suites Outex_TC_00010 and Outex_TC_00012. Our proposed descriptor $N I-L B P_{16,2}^{\text {riu } 2} / R D-$ 
Table 5: Classification accuracy (\%) of descriptor $N I / R D / C I$ for the three Outex test suites: training is done at just one rotation angle.

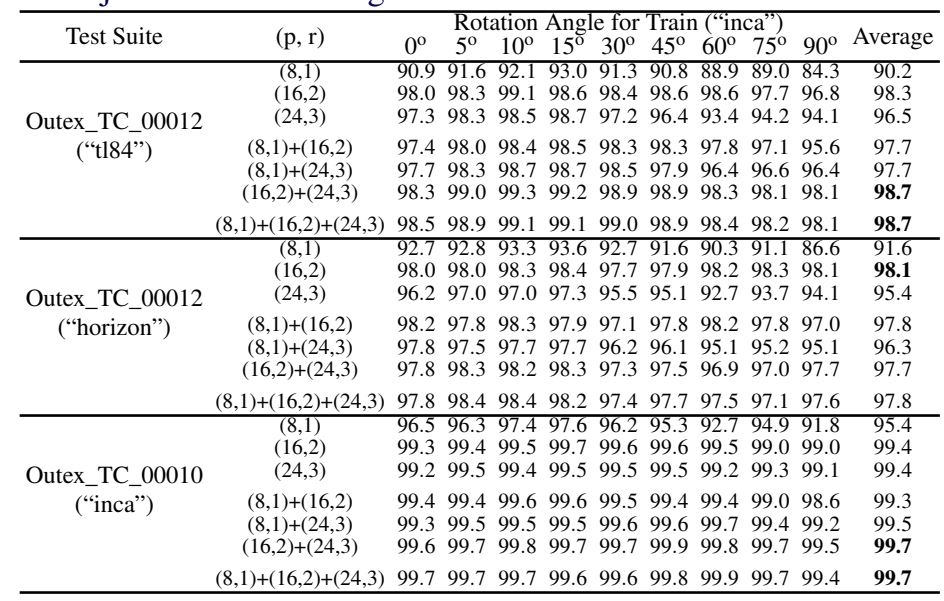

$L B P_{16,2}^{r i u 2} / C I-L B P$ produces consistently the best classification scores across all three test suites: $99.3 \%, 98.0 \%$ and $98.0 \%$ for Outex_TC_00010, Outex_TC_00012 "tl84" and Outex_TC_00012 "horizon" respectively, which are a considerable improvement over the best reported results of $97.9 \%, 90.2 \%$ and $87.2 \%$ on the corresponding test suite by Ojala et al. [5], especially for Outex_TC_00012 "t184" and "horizon", where the improvement is about $8 \%$ and $11 \%$ respectively.

Among individual descriptors, although $N I-L B P$ and $R D-L B P$ did not outperform $L B P$ or $V A R$, their combination significantly outperformed $L B P / V A R$. Consistent with the analysis in Section 2 that the intensity based descriptor $N I-L B P$ and the gradient based descriptor $R D-L B P$ are complementary, similar to the combination of RIFT and SIFT used by Zhang et al. [8].

Finally, we can see that $N I-L B P_{16,2}^{r i u 2} / R D-L B P_{16,2}^{r i u 2}$ and $N I-L B P_{16,2}^{r i u 2} / R D-L B P_{16,2}^{r i u 2} / C I-$ $L B P$ produce very robust classification performance in all three cases, in contrast to $L B P / V A R$, the performance of which decreases considerably in gray scale and rotation invariant texture classification. The excellent classification results demonstrate that $N I-L B P / R D-$ $L B P / C I-L B P$ is more stable for texture classification irrespective of imaging geometries of the illuminants affecting the appearance of local distortions caused by the tactile dimension of the textures.

Motivated by its excellent classification performance of $N I-L B P / R D-L B P / C I-L B P$, Table 5 presents extensive experiments on the three Outex test suites by varying the training angle. The results show robustness, especially for $N I-L B P_{16,2}^{r i u 2} / R D-L B P_{16,2}^{r i u 2} / C I-L B P$, and the improvement of results for multiresolution analysis over single resolution.

Fig. 4 compares the best scores achieved by our proposed method and those reported by competing state-of-the-art methods, which our approach outperforms. Whereas our proposed approach is training-free and involves simple computations, VZ-MR8 and VZ-Joint require a universal texton dictionary learning stage, followed by a histogram model learning stage. Our 5\%-7\% improvement over VZ-MR8 and VZ-Joint a likely a function of the limited training samples for learning the universal texton dictionary in VZ-MR8 and VZ-Joint. 


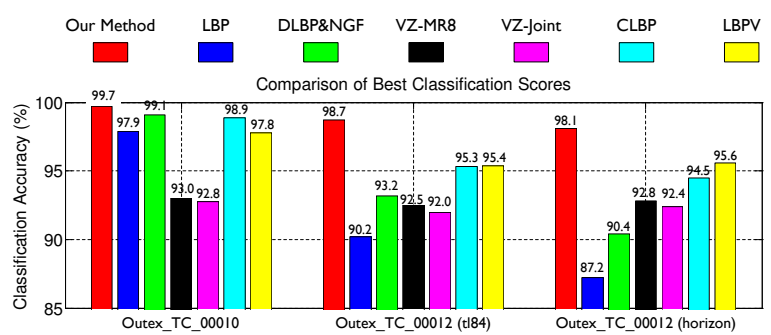

Figure 4: Comparing the best classification scores of our approach with various state-ofthe-art methods on all the three Outex test suites. All the results are as originally reported, except for those of VZ-MR8 and VZ-Joint, which are obtained by us using the exact same experimental setup as Varma and Zisserman did [2, 3]. For VZ-MR8 and VZ-Joint, 40 textons per class is used for building the universal texton dictionary.

Table 6: Left table: classification results (\%) of the proposed descriptors and the LBP on KTHTIPS2b. Right figure: Comparing the proposed approach with various state-of-the-art methods on KTHTIPS2b.

\begin{tabular}{c|ccc|ccc|ccc}
\hline \hline$(p, r)$ & \multicolumn{3}{|c|}{$(8,1)$} & \multicolumn{3}{|c|}{$(16,2)$} & \multicolumn{3}{|c}{$(24,3)$} \\
$N_{\text {train }}$ & 1 & 2 & 3 & 1 & 2 & 3 & 1 & 2 & 3 \\
\hline \hline$L B P$ & 48.1 & 54.2 & 56.8 & 50.5 & 55.8 & 59.1 & 49.9 & 54.6 & 57.8 \\
\hline \hline$N I$ & 46.1 & 48.7 & 52.3 & 37.6 & 41.2 & 44.4 & 39.0 & 42.6 & 45.4 \\
$R D$ & 48.1 & 54.2 & 56.9 & 44.0 & 49.4 & 52.1 & 38.5 & 42.7 & 45.2 \\
$R D / C I$ & 52.6 & 57.8 & 61.2 & 49.9 & 56.2 & 59.8 & 48.0 & 53.5 & 56.3 \\
$N I / C I$ & 47.1 & 53.1 & 56.4 & 46.5 & 50.5 & 53.0 & 44.8 & 48.8 & 51.6 \\
$N I / R D$ & 53.5 & 60.0 & 63.1 & 54.0 & 59.0 & 61.6 & 49.5 & 55.2 & 59.1 \\
$N I / R D / C I$ & 56.6 & 61.9 & 64.8 & $\mathbf{5 7 . 7}$ & $\mathbf{6 2 . 5}$ & 65.1 & 52.4 & 57.5 & 61.7 \\
\hline$(\mathrm{p}, \mathrm{r})$ & \multicolumn{3}{|c|}{$(8,1)+(16,2)$} & \multicolumn{3}{c|}{$(16,2)+(24,3)$} & \multicolumn{2}{c}{$(8,1)+(16,2)+(24,3)$} \\
$N_{\text {train }}$ & 1 & 2 & 3 & 1 & 2 & 3 & 1 & 2 & 3 \\
\hline$N I / R D / C I$ & 58.1 & 62.9 & 66.0 & 55.9 & 61.0 & 64.2 & 56.7 & 61.7 & 65.0 \\
\hline \hline
\end{tabular}

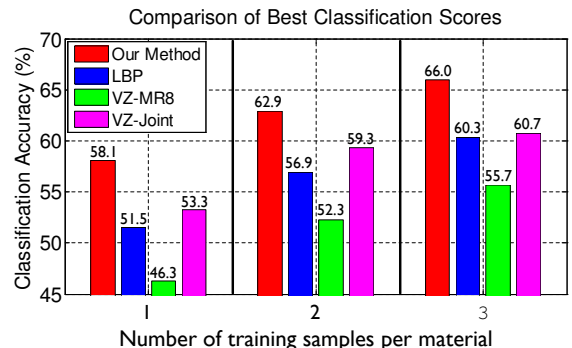

\subsection{Experiment \#2}

Image Data and Experimental Setup. The KTHTIPS2b database contains 11 different materials, each material with 4 different physical material samples, totaling 44 physical samples. The acquisition procedure for KTHTIPS2b has been described in more detail in [27].

For the experiments on KTHTIPS2b, we follow the similar training and testing scheme used in [21]. Each set of physical sample (108 images) can represent a testing or training set. We first perform experiments where only a single sample is available during training. All images of that sample are placed in the training set, and testing is subsequently performed on all images of all remaining samples. We also perform similar experiments with two and then three samples in the training set. Testing is always conducted only on unseen samples. All possible combinations of training sets are considered.

Experimental Results. As shown in Table 6, we compare our method with state-ofthe-art methods on the material categorization task of KTHTIPS2b textures. Note that all results from other methods are quoted directly from [21]. Likewise, one can find in the right side of Table 6 that our approach performs the best. It again confirms that our descriptor $N I-L B P / R D-L B P / C I-L B P$ extracts powerful discriminating features. We should bear in mind that the classification results of all the methods are obtained with a $1 \mathrm{NN}$ classifier, since we are focusing our attention on the effectiveness of the descriptors rather than on the capabilities of the classifier. However, using the more advanced support vector machine 
(SVM) classifier proposed by Caputo et al. [21] might improve the performance significantly.

\section{Conclusions}

This paper proposes a novel local texture descriptor, generalizing LBP. The proposed approach is computationally simple, training-free, and data-independent. Extensive experimental results show that the joint distribution of CI-LBP, NI-LBP and RD-LBP significantly outperform the conventional LBP approach and other state-of-the-art methods on Outex test suites. Results on KTHTIPS2b database also demonstrate the superior performance of the proposed approach in comparison with several state-of-the-art methods.

\section{References}

[1] M. Tuceryan and A.K. Jain, Texture analysis, in: C.H. Chen, L.F. Pau, and P.S.P. Wang (Eds.), Handbook Pattern Recognition and Computer Vision, World Scientific, Singapore, 1993, pp. 235-276.

[2] M. Varma and A. Zisserman, A statistical approach to texture classification from single images, Int. J. Comput. Vision 62 (1-2) (2005) 61-81.

[3] M. Varma and A. Zisserman, A statistical approach to material classification using image patches, IEEE Trans. Pattern Anal. Mach. Intell. 31 (11) (2009) 2032-2047.

[4] M. Calonder, V. Lepetit and P. Fua. Brief: binary robust independent elementary features, In Proc. of European conference on computer vision (ECCV), Crete, Greece, 2010.

[5] T. Ojala, M. Pietikäinen and T. Mäenpää, Multiresolution gray-scale and rotation invariant texture classification with local binary patterns, IEEE Trans. Pattern Anal. Mach. Intell. 24 (7) (2002) 971-987.

[6] T. Leung and J. Malik, Representing and recognizing the visual appearance of materials using three-dimensional textons, Int. J. Comput. Vision 43 (1) (2001) 29-44.

[7] L. Liu and P. Fieguth, Texture classification from random features, IEEE Trans. Pattern Anal. Mach. Intell. To appear.

[8] J. Zhang, M. Marszalek, S. Lazebnik and C. Schmid, Local features and kernels for classification of texture and object categories: a comprehensive study, Int. J. Comput. Vision 73 (2) (2007) 213-238.

[9] M. Crosier, L. D. Griffin, Using basic image features for texture classification, Int. J. Comput. Vision 88 (3) (2010) 447-460.

[10] S. Lazebnik, C. Schmid and J. Ponce, A sparse texture representation using local affine regions, IEEE Trans. Pattern Anal. Mach. Intell. 27 (8) (2005) 1265-1278.

[11] T. Randen and J. Husøy, Filtering for texture classification: a comparative study, IEEE Trans. Pattern Anal. Mach. Intell. 21 (4) (1999) 291-310. 
[12] T. Ojala, M. Pietikäinen and D. Harwood, A comparative study of texture measures with classification based on feature distributions, Pattern Recognit. 29 (1) (1996) 5159 .

[13] Y. Rodriguez and S. Marcel, Face Authentication Using Adapted Local Binary Pattern Histograms, in: Proceedings of the ECCV, Graz, Austria, 2006.

[14] T. Ahonen, A. Hadid, and M. Pietikäinen, Face description with local binary patterns: application to face recognition. IEEE Trans. Pattern Anal. Mach. Intell. 28 (12) (2006) 2037-2041.

[15] M. Pietikäinen, T. Nurmela, T. Mäenpää and M. Turtinen, View-based recognition of real-world textures, Pattern Recognit. 37 (2) (2004) 313-323.

[16] S. Liao, M.W.K. Law and A.C.S. Chung, Dominant local binary patterns for texture classification, IEEE Trans. Image Process. 18 (5) (2009) 1107-1118.

[17] Z. Guo, L. Zhang and D. Zhang, A completed modeling of local binary pattern operator for texture classification, IEEE Trans. Image Process. 9 (16) (2010) 1657-1663.

[18] M. Heikkilä, M. Pietikänen and C. Schmid. Description of interest regions with local binary patterns, Pattern Recognit. 42 (3) (2009) 425-436.

[19] J. Chen, S. Shan, C. He, G. Zhao, M. Pietikäinen, X. Chen and W. Gao, WLD: A robust local image descriptor, IEEE Trans. Pattern Anal. Mach. Intell. 32 (9) (2010) $1705-1720$.

[20] M. Unser, Sum and difference histograms for texture classification, IEEE Trans. Pattern Anal. Mach. Intell. 8 (1) (1986) 204-222.

[21] B. Caputo, E. Hayman, and P. Mallikarjuna, Class-specific material categorization, in: Proceedings of the ICCV, Beijing, 2005, pp. 1597-1604.

[22] T. Ahonen, M. Pietikäinen, Soft histograms for local binary patterns, in: Finnish Signal Processing Symposium, Oulu, Finland, 2007.

[23] X. Tan and B. Triggs, Enhanced local texture feature sets for face recognition under difficult lighting conditions, IEEE Trans. on Image Process. 19(6) (2010) 1635-1650.

[24] B. Caputo, E. Hayman, M. Fritz and J.-O. Eklundh, Classifying materials in the real world, Image and Vision Comput. 28 (1) (2010) 150-163.

[25] P. Brodatz, Texture: A Photographic Album for Artists and Designers. New York: Dover, 1966.

[26] Z. Guo, L. Zhang and D. Zhang, Rotation Invariant texture classification using LBP variance (LBPV) with global matching, Pattern Recognit. 43 (3) (2010) 706-719.

[27] P. Mallikarjuna, M. Fritz, A.T. Targhi, E. Hayman, B. Caputo, J.-O. Eklundh, The KTH-TIPS and KTH-TIPS2 databases, http://www. nada.kth. se/cvap/ databases/kth-tips/, 2006. 